\title{
Répondre aux besoins des jeunes filles à risque de mariage précoce et des adolescentes mariées au Burkina Faso
}

\author{
Sarah Engebretsen \\ Population Council \\ Gisele Kaboré \\ Population Council
}

Follow this and additional works at: https://knowledgecommons.popcouncil.org/departments_sbsr-pgy

Part of the Demography, Population, and Ecology Commons, Family, Life Course, and Society Commons, Gender and Sexuality Commons, and the International Public Health Commons How does access to this work benefit you? Let us know!

\section{Recommended Citation}

Engebretsen, Sarah and Gisele Kaboré. 2011. "Répondre aux besoins des jeunes filles à risque de mariage précoce et des adolescentes mariées au Burkina Faso," Promouvoir des transitions vers l'âge adulte saines, sécurisées et productives Resumen no. 9. New York: Population Council. 


\section{Répondre aux besoins des jeunes filles à risque de mariage précoce et des adolescentes mariées au Burkina Faso}

\section{Préparé par Sarah Engebretsen and Gisele Kabore}

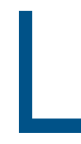
e Burkina Faso, pays enclavé d'Afrique de l'Ouest avec une population de 16,2 millions d'habitants, continue à connaître une croissance démographique rapide. Selon les prévisions démographiques, la population du Burkina Faso augmentera de 64 pour cent au cours des 15 prochaines années (PRB 2010). Cette croissance découle en partie de la jeune structure d'âge - le Burkina Faso est le troisième pays du monde où la population est la plus jeune, derrière le Niger et l'Ouganda, avec 46 pour cent de la population âgée de moins de 15 ans (PRB 2010) - ainsi que du mariage précoce et des naissances qui s'ensuivent. Le mariage précoce se produit fréquemment dans les zones rurales, où 62 pour cent des filles âgées de 20-24 ans sont mariées avant l'âge de 18 ans. En outre, le mariage avant l'âge de 15 ans peut atteindre 19 pour cent dans la région du Sahel (Population Council, 2009a).

Les traditions, les espérances familiales et le manque d'opportunités sont tous des éléments qui provoquent le mariage des filles avant l'âge légal de 17 ans. Cette situation limite leur capacité à terminer leur scolarité et à réaliser leur potentiel. Ces filles sont souvent la deuxième ou la troisième épouse d'un partenaire qui est sensiblement plus âgé qu'elles. Une fois mariées, elles sont confrontées à d'autres problèmes : elles ont tendance à être moins libres de leurs mouvements et ont des réseaux sociaux limités par rapport aux filles célibataires. L'on attend des filles nouvellement mariées qu'elles commencent à avoir des enfants presque immédiatement; elles donnent généralement naissance à leur premier enfant dans les 20 premiers mois suivant le mariage. Compte tenu de leur jeune âge, les adolescentes enceintes courent également risque de souffrir de complications pendant l'accouchement, y compris un travail prolongé et dystocique pouvant entraîner une fistule. Dans son rapport 2010,

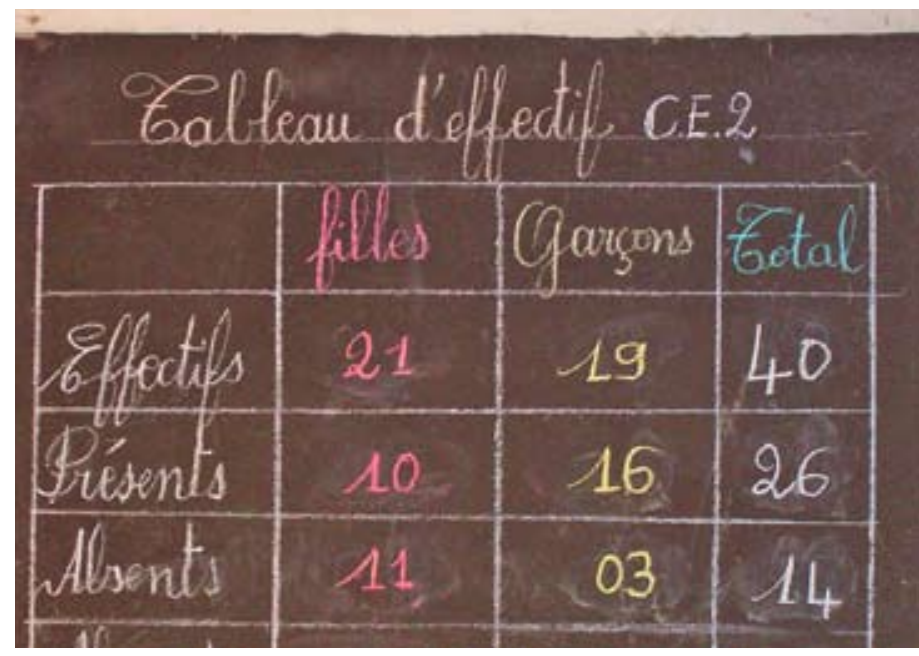

Ce registre de présence d'une classe d'enfants de 10 à 12 ans en milieu rural au Burkina Faso montre que plus de 50 pour cent des filles sont absentes de l'école. Le mariage précoce des adolescentes contribue à l'abandon scolaire.

State of the World's Mothers, Save the Children a classé le Burkina Faso parmi les pays les moins développés au monde au niveau de l'état du développement et de la santé des mères, illustrant à la fois le risque de grossesse et d'accouchement et le manque de soutien social aux les jeunes mères.

Malgré le nombre important de jeunes filles mariées et des filles à risque de mariage précoce dans les zones rurales $\mathrm{du}$ Burkina Faso, peu de programmes abordent la question du mariage précoce en s'engageant directement avec les filles et les dirigeants communautaires. En outre, le pays manque de services adaptés à 
leurs besoins. Sachant que les services de santé sont concentrés dans les zones urbaines, les distances à parcourir pour se rendre au centre de santé le plus proche représentent un obstacle de taille pour les filles qui vivent dans les zones rurales, en particulier celles dont la mobilité est limitée. Même si un centre de santé est accessible, les filles et les jeunes femmes doivent obtenir la permission de leur mari ou du chef de famille pour s'y rendre. S'il faut que les filles aient accès aux services de santé, les programmes de santé publique doivent s'attaquer à ces obstacles géographiques et sociaux.

\section{Le contexte du mariage précoce au Burkina Faso}

Au cours de la dernière décennie, le Population Council a mené des travaux de recherche qualitative et quantitative pour mieux comprendre les besoins et les expériences des filles à risque de mariage précoce et des jeunes filles mariées au Burkina Faso (Saloucou 2002 ; Saloucou et al 2003). Ces travaux ont montré que :

- La majorité des mariages étaient des mariages arrangés et les filles étaient souvent été données comme récompenses à la famille du marié. Dans les cas où les échanges de personnes n'avaient pas eu lieu simultanément, les jeunes filles étaient « dues » à la famille, sachant que ce type d'accord pouvait avoir lieu dès la naissance des fillettes. Une pratique moins fréquente au Burkina Faso consiste à échanger une jeune fille contre des biens et des services ou pour assurer la pérennité des liens entre les familles.

- Au cours de la période précédant le mariage, les filles reçoivent une instruction sur les travaux ménagers, la garde des enfants et le comportement approprié (soumission) à adopter envers leurs maris et beaux-parents. Elles subissent des restrictions encore plus sévères vis-à-vis de leur liberté de mouvement et de leurs relations.

- Les adolescentes ne sont pas aussi libres de leurs mouvements que leurs homologues masculins, et la liberté de mouvement des filles mariées se limite à se rendre aux endroits qui leur permettent de s'acquitter des tâches ménagères et de garder les enfants.

- Les adolescentes mariées n'ont pas recours aux centres de santé.

- Les garçons ont accès aux établissements récréatifs et de loisirs, ce qui leur donne l'occasion de faire des rencontres avec des pairs et d'obtenir des informations sur la santé ; les filles se voient refuser un accès comparable aux réseaux sociaux et aux informations.

- Tant les filles mariées que les filles non mariées ont un besoin urgent d'informations et de ressources sur la santé, ainsi que de l'accès aux centres de santé.
La recherche en pratique

Les travaux de recherche du Population Council ont révélé que les filles mariées burkinabé souffraient de contraintes liées à leur temps libre, à leur liberté de mouvement et au manque d'accès aux centres de santé. Le seul moyen d'intervenir dans cette population est de les toucher à leur domicile. Ces résultats ont orienté l'élaboration d'une intervention novatrice connue sous le nom de Programme des « mèreséducatrices ». Le rôle principal de mères-éducatrices a été de fournir des informations et un soutien aux adolescentes mariées au cours de leur première grossesse et accouchement, et d'apporter des suppléments de vitamine $\mathrm{A}$ et de fer à celles qui étaient enceintes. Compte tenu de l'isolement des adolescentes mariées, les mères-éducatrices voyageaient en paires (au moyen de bicyclettes fournies par le projet) au domicile des filles vivant dans les zones reculées ; une mèreéducatrice discutait avec le chef de famille en lui expliquant le programme et en fournissant des informations, alors que l'autre mère-éducatrice tentait d'établir un dialogue avec l'adolescente mariée. Pour accroître l'utilisation des centres de santé locaux, les mères-éducatrices ont joué un rôle de liaison entre les adolescentes mariées et le personnel des

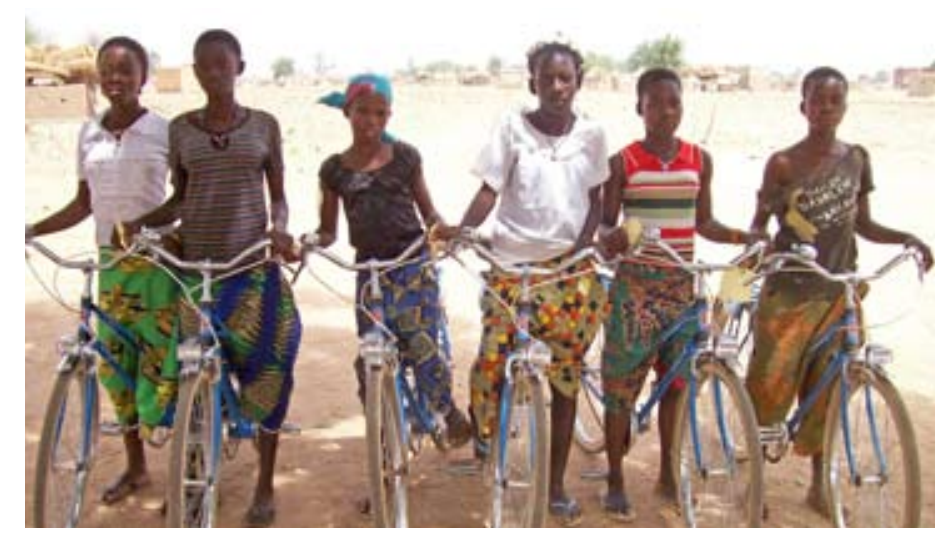

Les filles scolarisées ont obtenu des bourses et des bicyclettes pour les encourager à poursuivre leurs études.

centres de santé. En plus d'accompagner les filles aux centres pour des visites prénatales et des séances de formation, les mères-éducatrices ont également aidé à sensibiliser les travailleurs de santé aux besoins spécifiques et à la vulnérabilité des jeunes filles mariées.

Le projet des mères éducatrices a démontré que l'amélioration de la santé sexuelle et reproductive des adolescentes impliquait de lutter contre la pratique du mariage précoce, tout en fournissant un appui aux filles mariées. Pour répondre à cette question, le Population Council s'est associé avec l'UNFPA, l'UNICEF et le Ministère de l'Action sociale et de la Solidarité nationale dans le cadre d'un projet pilote intitulé L'élimination de mariage des enfants au Burkina Faso : Un plan de protection, de renforcement et d'action communautaire. Ce projet visait à renforcer la sécurité physique des filles à risque de mariage ainsi que 


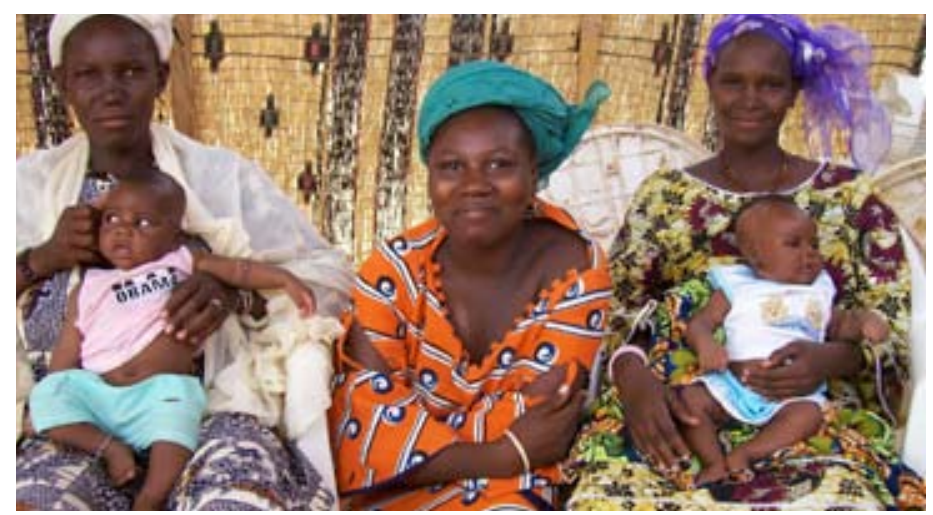

Trois mères-éducatrices à Dori (région du Sahel) qui ont servi de tutrices et des filles qui ont été informées sur les risques liés au mariage précoce et sur la santé reproductive.

celles qui sont déjà mariées. Le projet a souligné le rôle de la communauté pour appuyer et plaider en faveur du retard de l'âge du mariage. Le projet s'est déroulé dans 24 villages à travers cinq régions (CentreSud, Centre-Est, Est, Centre-Nord et Sahel).

Le programme a recruté des filles scolarisées et non scolarisées âgées de 10 à 19 ans qui risquaient de se marier ou qui étaient déjà mariées. Les filles qui étaient encore scolarisées ont obtenu des bourses et des bicyclettes pour poursuivre leurs études, bien qu'elles ne représentent qu'une très faible proportion du nombre total des filles incluses dans le programme (143 sur 4000 ). La majorité des filles ont reçu des fonds pour commencer une activité génératrice de revenus.

Ce programme pilote a recruté des mères-éducatrices pour servir de tutrices aux jeunes filles à risque de mariage précoce. Pour être admissibles, les mères-éducatrices devaient avoir entre 15 et 19 ans, être mariées avec au moins un enfant et être bien considérées dans leurs communautés. Les mères-éducatrices ont été formées aux droits

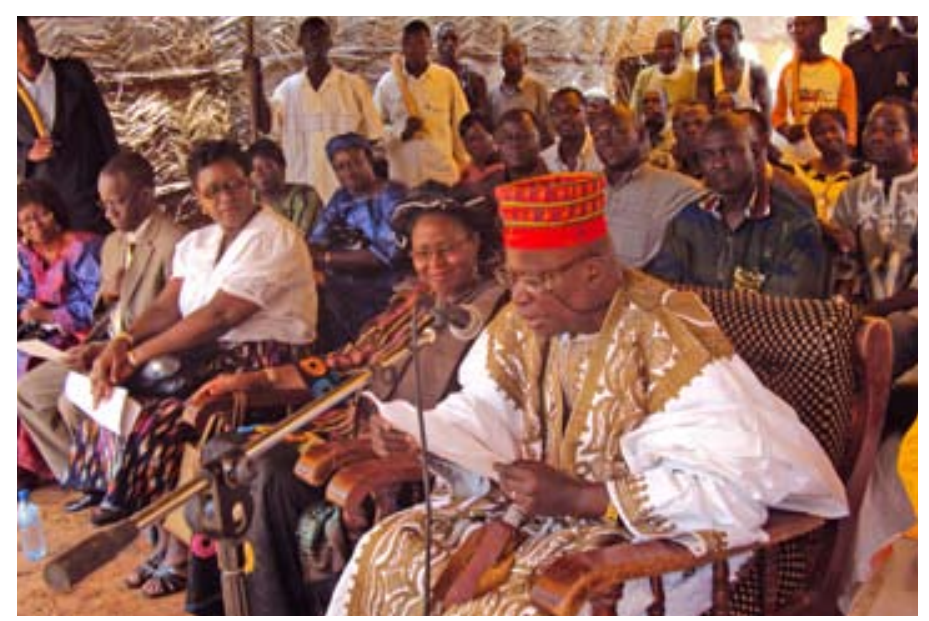

Séance de plaidoyer avec le roi de Boussouma encourageant le respect de l'âge légal du mariage, qui est de 17 ans pour les femmes et de 20 ans pour les hommes. de l'homme, aux compétences de vie, à la santé sexuelle et reproductive et aux moyens de subsistance. Trois fois par mois, elles dispensaient des séances d'éducation aux filles sur plusieurs thèmes clés en lien avec le mariage précoce (par exemple, la planification familiale, les soins prénatals, les IST, le VIH, les mutilations génitales féminines et la fistule). Elles ont en outre effectué des visites à domicile et orienté les filles qui avaient des besoins de santé sexuelle et reproductive vers des établissements de santé. Les mères-éducatrices ont reçu une aide financière pour démarrer une activité génératrice de revenus comme la couture ou l'élevage d'animaux.

L'existence d'un environnement social favorable est essentielle pour changer les normes sociales et lutter contre le mariage précoce. L'équipe du projet a fait participer directement les personnes chargées de prendre les décisions au nom des adolescentes, tels que les pères,

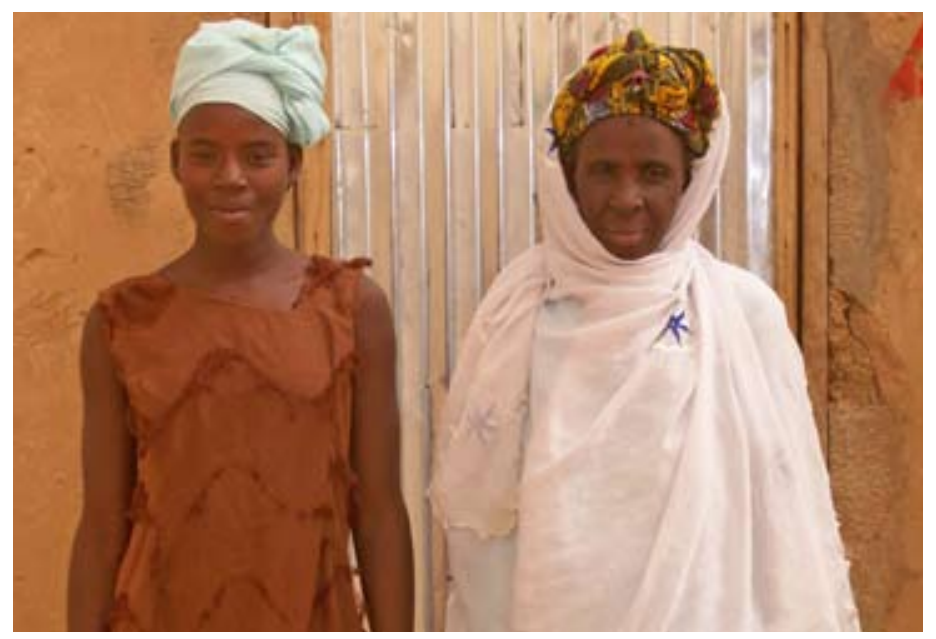

Cette jeune femme a évité un mariage avec un homme âgé de près de trois fois son âge parce que sa mère a contesté la pratique traditionnelle.

les maris, les belles-mères et les autres membres de la famille. Des équipes en communication composées de membres de la communauté dans chaque site ont effectué des visites en porte à porte ; mené des séances de sensibilisation et conduit des activités de plaidoyer sur la lutte contre le mariage précoce et l'importance de la scolarisation avec les parents des adolescentes, les chefs traditionnels et les chefs religieux ; elles ont informé les animateurs de la communauté des actions réalisées ou des services fournis par le Ministère de l'Action sociale et joué le rôle de médiateurs en cas de menace de mariage précoce. Les chefs traditionnels et religieux ont renforcé les messages diffusés par le programme et ont été considérés comme des acteurs clés dans la lutte contre les mariages précoces dans leurs communautés. Par exemple, le roi de Boussouma a déclaré qu'après avoir pris conscience de l'ampleur du mariage précoce dans sa communauté et de ses conséquences, lui-même ainsi que la communauté s'engageaient 
pleinement à « lutter résolument et avec efficacité contre les mariages précoces, sous toutes leurs formes, à travers le royaume du roi pour s'assurer que ce phénomène ne soit plus qu'un mauvais souvenir dans les années à venir ». Le Grand Imam de Dori a déclaré que « les conséquences liées à la pratique du mariage précoce sont désormais connues ». Dans les petits villages, les chefs religieux doivent accroître leurs efforts de plaidoyer. Nous [les imams] pouvons contribuer à cet effort, parce que même si dans la pratique les mariages précoces ont diminué dans les villes, il est encore très répandu dans les villages ». Les efforts de plaidoyer ont suscité l'intérêt de la communauté pour lutter contre la pratique du mariage précoce et le programme a fait l'objet d'une demande par les communautés proches des sites d'intervention.

Les connaissances, les attitudes, les pratiques et les perceptions liées au mariage précoce et à la vie de jeunes filles mariées ont été évaluées à l'aide d'études initiales et finales dans les cinq régions d'intervention. Pour chercher les effets possibles sur la communauté, des entretiens ont été menés auprès d'environ 1700 ménages et 1400 adolescentes âgées de 10 à 19 ans au départ et de 10 à 21 ans en fin d'étude. Les résultats ont montré une amélioration du niveau de connaissances des chefs de famille et des adolescentes sur l'âge minimum légal du mariage, une amélioration des connaissances des adolescentes sur la fistule obstétricale et les moyens d'éviter une grossesse et sur l'utilisation des services de santé sexuelle et reproductive, en particulier pour l'aide à l'accouchement.

\section{Enseignements tirés}

Les travaux de recherche du Population Council montrent qu'il est possible d'identifier, d'atteindre et de soutenir les adolescentes mariées et les personnes à risque de mariage précoce dans des circonstances traditionnelles-même celles qui vivent dans des communautés rurales, éloignées et pauvres en ressources. Les interventions du Population Council ont mobilisé des groupes influents de jeunes femmes leaders comme mères-éducatrices. Ces jeunes mères ont encadré les filles et ont travaillé avec les prestataires de santé afin d'assurer que les services étaient ciblés sur les besoins des adolescentes. Ces travaux montrent également qu'il est possible de mobiliser les communautés et d'accroître la sensibilisation sur les dangers du mariage précoce. Ce type d'engagement communautaire constitue la base pour transformer les normes sociales et la communication sur les changements de comportement.

\section{Bibliographie et publications connexes}

Amin, Sajeda and Ashish Bajracharya. 2011. "Assessing the costs of marriage: Marriage transactions in the developing world." Transitions to Adulthood Brief No. 37. New York : Population Council.

Brady, Martha, Lydia Saloucou, et Erica Chong. 2007. "Girls' adolescence in Burkina Faso: A pivot point for social change." New York : Population Council.

Population Council. 2009a. "The Adolescent Experience In-Depth: Using Data to Identify and Reach the Most Vulnerable Young People: Burkina Faso 2003." New York.

Population Council. 2009b. "Étude quantitative sur le mariage précoce et le vécu des adolescents dans la zone d'intervention du projet : état des lieux et perspectives." Ouagadougou.

Population Reference Bureau (PRB). 2010. "2010 World population data sheet." Washington, DC. <http://www.prb.org/Publications/ Datasheets/2010/2010wpds.aspx>. Consulté le 6 mai 2011.

Saloucou, Lydia. 2002. "Étude diagnostique du vécu des filles mariées et de leurs besoins en opportunités économiques et en santé de la reproduction." Ouagadougou : Population Council. Rapport non publié.

Saloucou, Lydia, Ida Nikyema, Cyrille Compaore, et Salomon Yameogo. 2003. "Itinéraires thérapeutiques des adolescents en grossesse ou première fois parents : Gaongo, Nafbanka, Natiaboani et Koaré." Ouagadougou : Population Council.

Save the Children. 2010. "State of the world's mothers." Westport, CT. <http:// www.savethechildren.net/alliance/what_we_do/every_one/news.html>. Consulté le 13 janvier 2011.

\section{Donateurs}

Le gouvernement du Japon par le biais du Fonds des Nations unies pour la sécurité humaine, le Ministère britannique du développement international (DFID), la Fondation Bill \& Melinda Gates, le Fonds des Nations unies pour la population (UNFPA), la Fondation des Nations unies.

\section{Organisations partenaires}

UNICEF

UNFPA

Ministère de l'Action sociale et de la Solidarité nationale

Ministère de la Santé

Direction de l'Hygiène Publique et de l'Éducation à la Santé

Direction de la Santé et de la Famille

Direction générale de la Coopération

Ministère de l'Enseignement de base et de l'Alphabétisation

Ministère des Enseignements secondaire, supérieur et de la Recherche

Ministère de la promotion de la Femme

Ministère de Promotion des droits de l'Homme

Secrétariat permanent / Conseil national pour la population

\section{Population Council}

Le Population Council fait évoluer les perspectives sur les questions essentielles de santé et de développement. Nous cherchons à comprendre les causes et les conséquences de l'inégalité entre les sexes et des disparités sociales qui s'installent au cours de l'adolescence. Nous fournissons des données en vue d'améliorer les programmes et les politiques mis en place sur le terrain qui permettent d'assurer des transitions efficaces et productives vers la vie adulte dans les pays en développement. www.popcouncil.org

(c) 2011 The Population Council, Inc.

4 - Rendez-vous sur www.popcouncil.org/publications/serialsbriefs/TABriefs.asp pour consulter tous les bulletins d'information de la série Promoting healthy, safe, and productive transitions to adulthood [Promouvoir des transitions saines, sécurisées et productives vers l'âge adulte]. 